\title{
Significance of the Cerebellopontine Cistern Cross-Sectional Area and Trigeminal Nerve Anatomy in Trigeminal Neuralgia: An Anatomical Study Using Magnetic Resonance Imaging
}

\author{
Aylin GUNESLI ${ }^{1}$, Kadir TUFAN² \\ ${ }^{1}$ Baskent University, Department of Radiology, Ankara, Turkey \\ Baskent University, Department of Neurosurgery, Ankara, Turkey \\ This study has been presented as an poster presentation at the European Society of Neuroradiology $41^{\text {st }}$ Annual Meeting between 19 and 23 September \\ 2018 at Rotterdam, The Netherlands
}

Corresponding author: Kadir TUFAN kadirtufan@hotmail.com

\section{ABSTRACT}

AIM: To evaluate the relationship between trigeminal neuralgia (TN) and potential magnetic resonance imaging (MRI)-related measurements in patients with TN.

MATERIAL and METHODS: Retrospective analysis of 104 patients with TN was performed. MRI studies of 98 healthy controls were included in the study to compare the parameters with TN patients' measurements. MRI measurements of cerebellopontine cistern (CPC) cross-sectional area, trigeminal-pontine angle (TPA) width, and trigeminal nerve cisternal segment length and thickness were assessed on both symptomatic and asymptomatic sides using 1.5T MRI with constructive interference in steady-state sequences. The images were interpreted by two radiologists blinded to the affected sides of the patients.

RESULTS: There were significant differences between the symptomatic and asymptomatic sides in terms of mean trigeminal nerve length $(8.8 \pm 2.34 \mathrm{~mm}$ vs. $9.39 \pm 2.29 \mathrm{~mm}$; respectively, $\mathrm{p}=0.001)$ and thickness $\left(20.9 \pm 9.6 \mathrm{~mm}^{2} \mathrm{vs} .25 \pm 9.98 \mathrm{~mm}^{2}\right.$, respectively; $\mathrm{p}<0.001)$. The median cerebellopontine cistern cross-sectional area was considerably lower on the symptomatic side compared with the asymptomatic side [201 $\mathrm{mm}^{2}$ (interquartile range=93) vs. $224.5 \mathrm{~mm}^{2}$ (interquartile range $=77$ ), respectively; $\mathrm{p}<0.001$ ] There were no significant differences between the trigeminal-pontine angle width on either side (38.32 \pm 10.38 vs. $38.78 \pm 10.9$, respectively; $p=0.679$ ). There were no statistically significant differences between the right and left sides regarding these parameters in the control group.

CONCLUSION: Smaller CPC cross-sectional area, trigeminal nerve length, and trigeminal nerve thickness on MRI were demonstrated to commonly exist on the symptomatic side in patients with TN. We suggest that this narrow space may increase the risk of vascular compression on the nerve.

KEYWORDS: Trigeminal neuralgia, Trigeminal nerve length, Trigeminopontine angle, Cerebellopontine cistern, Magnetic resonance imaging

\section{INTRODUCTION}

T The trigeminal neuralgia (TN) presents with a sudden onset of severe, unilateral, and paroxysmal pain including one or more branches of the trigeminal nerve $(13,15,20)$. The ophthalmic branch (V1), the maxillary branch (V2), and the mandibular branch (V3) was involved in 17\%, $67 \%$, and $50 \%$ of the patients, respectively. TN is a unique type of neuropathic pain and was first described in 1773 by John Fothergill. It has a prevalence of 200 in 100,000 and an incidence of 2.7 in 100,000 per year $(6,17)$.

Neurovascular compression (NVC) syndrome is described as mechanical compression of cranial nerves by blood vessels. Arteries cause symptomatic NVC syndrome more frequently than veins (6). The anterior inferior cerebellar artery, superior 
cerebellar artery, and basilar artery commonly compress vessels to the trigeminal nerve (4). TN is the most common type of NVC syndrome (10). The region from which the trigeminal nerve exits the brain stem and reaches Meckel's cave is the most common site of NVC. In addition to the secondary causes of TN, such as tumors, multiple sclerosis, arteriovenous malformations, and trauma, NVC is the most common causative factor of classic or idiopathic TN $(1,15)$. The exact cause underlying NVC is unknown; however, some anatomical variations have been described that have a tendency toward TN. Vascular variations, such as dolichoectasia, an aberrant vessel loop, and tortuosity are the most reported and wellknown anatomical variations $(12,13,15)$. Other than vascular variations, smaller cerebellopontine cistern (CPC) crosssectional area (CPCA) and sharper trigeminal-pontine angle (TPA) were reported to be associated with increased risk for NVC $(11,21)$. Trigeminal atrophy is a common morphological finding of $\mathrm{TN}$ and is often accompanied by distortion, groove formation, and deviation. It can be assessed by measuring the area and length of the cisternal segment of the trigeminal nerve $(7,11)$.

Magnetic resonance imaging (MRI) is an efficient modality for imaging posterior fossa structures, and it can help exclude the secondary causes of TN (9). In an MRI study conducted by Ha et al., a sharp TPA was associated with increased TN risk (11). Park et al. showed that nerve atrophy and small CPCA were related to TN (19). High-resolution MRI has allowed radiologists to achieve a detailed image of the cisternal and Meckel's cave segments of the trigeminal nerves $(5,10)$. As a standard sequence for imaging TN, high-resolution threedimensional (3D) T2-weighted steady-state free precession sequences, such as FIESTA or 3D-CISS, provide high contrast and high spatial resolution between the cerebrospinal fluid and the neurovascular tissues $(5,10)$. Blitz et al. hypothesized that adding intravenous contrast material to 3D-CISS sequences, which appear to be T2-weighted but have both T2- and T1-weighting, would improve the contrast between enhanced vessels and adjacent unenhanced trigeminal nerve roots (5). In addition, 3T and higher-field MRI images provide increased diagnostic sensitivity and specificity for significant improvement in different neurologic disorders, such as TN (10). In this study, we aimed to analyze the relationship between TN and several relevant MRI-related measurements, such as the CPC cross-sectional area, TPA width, and the trigeminal nerve cisternal segment cross-sectional area and length, in patients with TN.

\section{MATERIAL and METHODS}

\section{Patients}

Our study sample included 106 patients with classical TN who were diagnosed at our center between 2011 and 2018. TN was diagnosed according to the International Classification of Headache Disorders (2004), which includes persistent symptoms for six months and resistance to medical therapy. Patients with secondary $\mathrm{TN}$ and bilateral symptoms were excluded.

A total of $98 \mathrm{MRI}$ studies of healthy individuals were evaluated to serve as a control group. These MRI studies were previously performed for reasons that were not related to cerebellopontine angle pathologies.

This retrospective study was approved by the Baskent University Ethics Committee. Informed consent was obtained from all the patients.

\section{Imaging and Measurement Procedures}

All patients were examined with a single MR scanner (1.5T MRI System Magnetom Avanto; Siemens, Erlangen, Germany). High-resolution T2-weighted CISS sequences with a section thickness of $0.6 \mathrm{~mm}$ were used to measure the related parameters (repetition time: 1,000 ms; echo time: $263 \mathrm{~ms}$; Acq Matrix: $320 \times 320 \mathrm{~mm}$; voxel size: $0.6 \mathrm{~mm} \times 0.6 \mathrm{~mm} \times 0.6$ $\mathrm{mm})$. The images were interpreted by 2 radiologists who were blinded to the affected side of the patients. The CPCA was measured from the basilar artery to the cerebellar floccules and was limited by the posterior surface of the petrous bone (Figure 1A, B). The trigeminal nerve length was measured from the zone where the nerve exits the pons to the entry point of Meckel's cave (Figure 2A, B). The cross-sectional area of
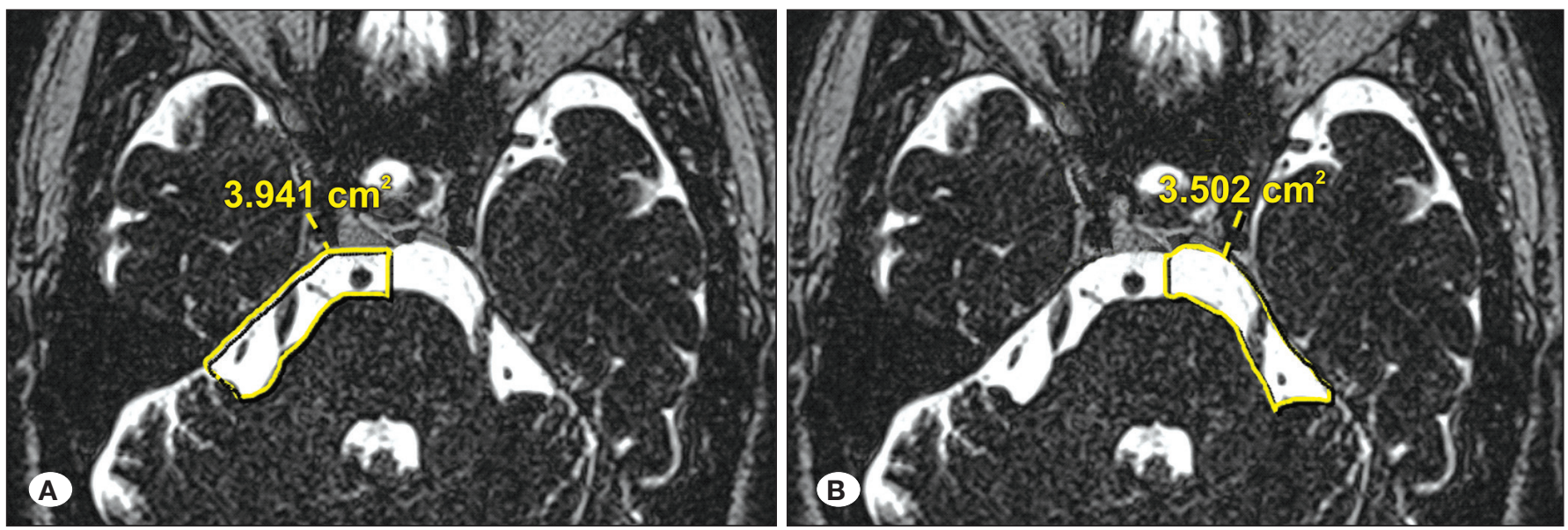

Figure 1A, B: 51-year-old male with 3-year history of left sided trigeminal neuralgia. Cross-sectional area of the cerebellopontine cistern. The area is smaller on the symptomatic side. 

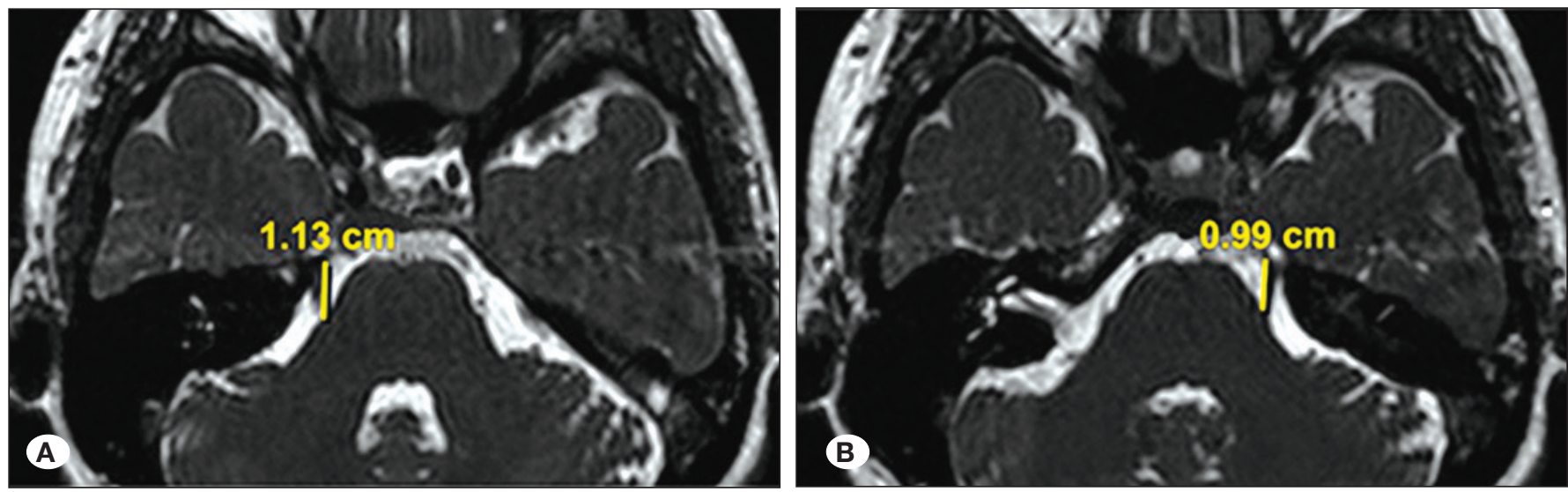

Figure 2 A, B: 55-year-old male with a 4-year history of left sided trigeminal neuralgia. Axial images of trigeminal root at the level of Meckel's cave. Measurements of the length of the trigeminal nerve. The trigeminal nerve length is shorter on the symptomatic left side.
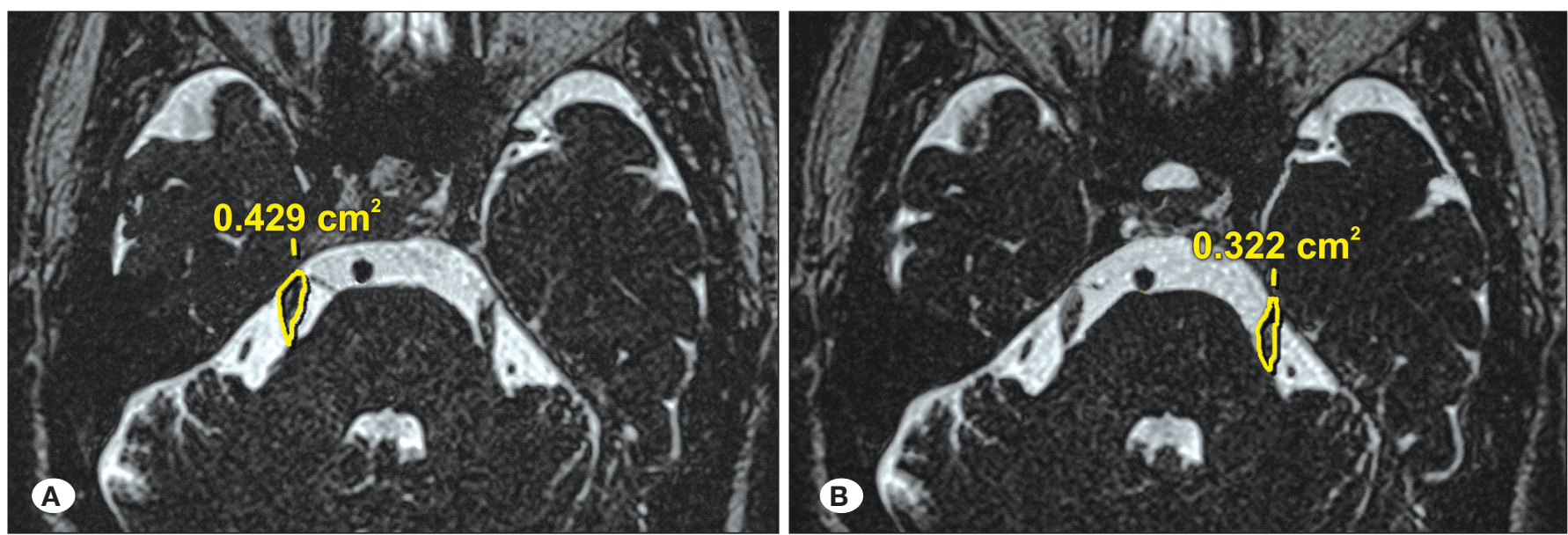

Figure 3 A, B: 50-year-old female who had left sided trigeminal neuralgia for 8 years. Cross-sectional area of the trigeminal nerve area at the level of Meckel's cave. The measurement is smaller on the symptomatic left side.

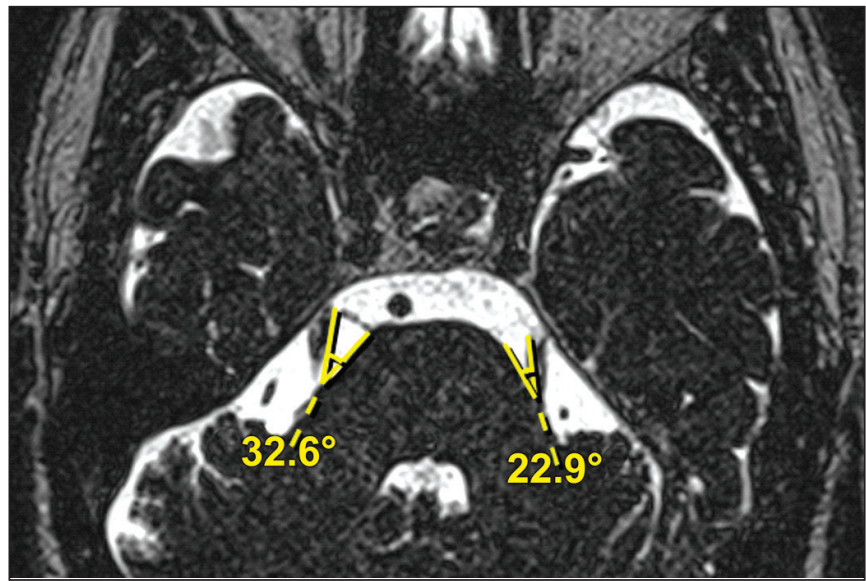

Figure 4: 51-year-old male with a 3-year history of left sided trigeminal neuralgia. MRI is showing delineation of the angle between the trigeminal nerve and anterior surface of the pons. the cisternal segment of the trigeminal nerve was measured in axial images (Figure 3A, B). TPA was defined as the angle between the medial margin of the trigeminal nerve and the anterior surface of the pons (Figure 4).

\section{Statistical Analysis}

Continuous variables were tested for a normal distribution with the Kolmogorov-Smirnov test. The results for normally distributed variables were expressed as the mean \pm standard deviation and non-normally distributed variables were expressed as the median and interquartile range (IQR). Categorical variables were shown as absolute values and percentages. A paired samples t-test or independent samples t-test was used to compare the two groups with respect to the variables with a normal distribution and homogeneous group variance as appropriate (paired samples t-test for dependent variables, independent samples t-test for independent variables). Variables that had a non-homogenous distribution were compared with a Wilcoxon signed-rank test. Categorical variables of independent samples were compared with a chi- 
squared test and categorical variables of dependent samples were compared with a McNemar test and $\mathrm{p}<0.05$ was considered statistically significant. Statistical analyses were performed using SPSS version 20 (SPSS, Chicago, IL, USA).

\section{RESULTS}

\section{TN Group}

A total of 106 patients with TN were included in the study. The mean age was $57.6 \pm 14.7$ years (mean \pm standard deviation, range: $20-89$ years), and $35.8 \%$ of the population were male (38/126). Table I shows the comparison of the trigeminal nerve cross-sectional area and length, TPA, and CPCA measurements between the symptomatic and asymptomatic sides. The mean trigeminal nerve length was significantly smaller on the symptomatic side $(p=0.001)$. The trigeminal nerve area on the symptomatic side was significantly smaller than on the asymptomatic side $(p<0.001)$. The mean CPCA was also smaller on the symptomatic side than on the asymptomatic side $(p<0.001)$. However, there were no significant differences in the TPA values between the 2 sides $(p=0.679)$. NVC was detected in $79 \%$ of the patients on the symptomatic side, whereas detection on the asymptomatic side was $36 \%(p<0.001)$.

\section{Control Group}

A total of 98 healthy controls were included in the study to compare the parameters with measurements in patients with TN. The baseline clinical characteristics, such as age and sex, were similar for the control and TN groups. There was no statistically significant difference in the trigeminal nerve length, trigeminal nerve area, TPA, and CPCA between right and left sides in the control group (Table II). When evaluating the MRI studies in the healthy control group, NVC was detected in $26 \%$ of the individuals.

\section{Comparison of TN and Control Groups}

Only $26 \%$ of the individuals in the control group had NVC and the NVC ratio was much higher in the patient group (79\%, $\mathrm{p}<0.001$ ).

In the comparison of the trigeminal nerve cross-sectional area and length, TPA, and CPCA measurements for the right and left side, there was a statistically significant difference in the TN group. However, these parameters were similar between the right and left sides in the control group.

\section{DISCUSSION}

The main pathophysiology of classical TN has been a matter of continuous debate. TN diagnosis is typically determined clinically. MRI is mainly used to determine the neurovascular contact and to exclude secondary TN (2).

It is well-known that NVC can also be seen in healthy individuals. Without clinical evaluation, MRI has a low diagnostic specificity for TN $(5,6)$. However, the incidence of NVC detection with MRI is much higher in TN patients on the symptomatic side. In the present study, we found NVC in $79 \%$ of the patients with TN and $26 \%$ of the individuals in the control group.

The relationship between trigeminal nerve atrophy and TN as well as surgical series reporting smaller measurements for the nerve on the affected side have convinced investigators to use $\mathrm{MRI}$ measurements as diagnostic parameters for TN $(8,16)$. A smaller trigeminal nerve, CPCA, and TPA width, which are related to NVC and nerve atrophy, are the MRI parameters

Table I: Comparison of the Trigeminal Nerve Area, Length, Trigeminal-Pontine Angle, and Cerebellopontine Cistern Cross-Sectional Area Measurements

\begin{tabular}{lccc}
\hline & Symptomatic side & Asymptomatic side & p \\
\hline Trigeminal nerve area $\left(\mathrm{mm}^{2}\right)$ & $20.9 \pm 9.6$ & $25 \pm 9.98$ & $<0.001$ \\
\hline Trigeminal nerve length $(\mathrm{mm})$ & $8.8 \pm 2.34$ & $9.39 \pm 2.29$ & $\mathbf{0 . 0 0 1}$ \\
\hline TPA $\left(^{\circ}\right)$ & $38.32 \pm 10.38$ & $38.78 \pm 10.9$ & 0.679 \\
\hline CPCA $\left(\mathrm{mm}^{2}\right)$ & $201(\mathrm{IQR}=93)$ & $224.5(\mathrm{IQR}=77)$ & $<0.001$ \\
\hline
\end{tabular}

CPCA: Cerebellopontine cistern cross-sectional area, IQR: Interquartile range, TPA: Trigeminal pontine angle.

Table II: Comparison of Trigeminal Nerve Area, Length, Trigeminal-Pontine Angle and Cerebellopontine Cistern Cross-Sectional Area Measurements in the Control Group

\begin{tabular}{lccl}
\hline & Right side & Left side & p \\
\hline Trigeminal nerve area $\left(\mathrm{mm}^{2}\right)$ & $28.08 \pm 7.97$ & $27.49 \pm 8.56$ & 0.127 \\
\hline Trigeminal nerve length $(\mathrm{mm})$ & $10.48 \pm 1.63$ & $10.59 \pm 1.88$ & 0.38 \\
\hline TPA $\left(^{\circ}\right)$ & $36.7 \pm 8.96$ & $36.6 \pm 7.87$ & 0.862 \\
\hline CPCA $\left(\mathrm{mm}^{2}\right)$ & $200(\mathrm{IQR}=63)$ & $215(\mathrm{IQR}=53)$ & 0.268 \\
\hline
\end{tabular}

CPCA: Cerebello pontine cistern cross-sectional area, IQR: Interquartile range, TPA: Trigeminal pontine angle. 
of diagnostic interest $(7,11)$. Studies have shown that certain morphological changes, including axonal loss, axonopathy, demyelination, dysmyelination, residual myelin debris, and collagen deposition, can occur following chronic physical stress and may lead to trigeminal nerve atrophy in patients with TN (9). As for other indicators of nerve atrophy, a smaller trigeminal nerve length was shown to occur more frequently on the symptomatic side of patients with TN (19).

Erbay et al. reported that the nerve diameter and crosssectional area were $20 \%$ and $28 \%$ smaller on the symptomatic side, respectively, according to the asymptomatic side. In their study of 36 patients with TN, nerve area was significantly smaller on the affected side compared with the unaffected side $\left(4.50 \pm 1.75 \mathrm{~mm}^{2}\right.$ and $6.28 \pm 2.19 \mathrm{~mm}^{2}$, respectively; $\left.\mathrm{p}=0.0001\right)$, which is consistent with our results $\left(20.9 \pm 9.6 \mathrm{~mm}^{2}\right.$ vs. $25 \pm$ $9.98 \mathrm{~mm}^{2}$, respectively; $\left.p<0.001\right)$ (9). Horinek et al. revealed that the volume of the involved nerve was $28 \%$ smaller than the unaffected nerve in the 18 patients with TN in their study; however, only 7 of the 18 patients demonstrated nerve atrophy on MRI (14). According to Antonini et al., nerve atrophy and displacement were highly specific (97\%). Moreover, when REZ contact and nerve atrophy coexisted, both specificity and positive predictive value increased to $100 \%$. However, MRI has low selectivity because neurovascular contact might be detected in some asymptomatic individuals (3). Parise et al. measured the cross-sectional CPCA bilaterally utilizing axial T2 weighted, 3D CISS sequences (18). The CPCA was found to be significantly smaller on the side in which the trigeminal nerve was affected. Rasche et al. also reported that a smaller CPCA may be associated with an increased incidence of NVC between the trigeminal nerve and surrounding vessels (21). These reports were consistent with our study. Our results clearly demonstrated that the MRI measurements of trigeminal nerve length, trigeminal nerve area, and CPCA were all significantly smaller on the symptomatic side of the patients with TN. In terms of TPA measurements, there were no significant differences between the symptomatic and asymptomatic sides in our patients, which is opposite to the findings of $\mathrm{Ha}$ et al. (11).

There are some limitations in our study. First, common to any retrospective analysis at a single institution, some unpredictable biases may have altered our results. Second, the manual measurements of MRI-related parameters may have caused inter-observer variability. Third, measurements obtained using 1.5T MRI may have diminished the reliability of the results, compared to studies using $3 T$ MRI. However, the use of a larger patient cohort than in most contemporary studies and the simultaneous evaluation of 4 different parameters in the same cohort appear to be the strengths of the current study.

\section{CONCLUSION}

Although symptoms and clinical examinations are the established steps of the diagnostic algorithm used in patients with TN, evaluations of MRI-related measurements, such as the CPCA and the area and length of the trigeminal nerve, may enhance TN diagnosis. Smaller CPC cross-sectional area, trigeminal nerve lengths, and trigeminal nerve thickness on MRI, were demonstrated to commonly exist on the symptomatic side in patients with TN. We suggest that this narrow space may increase the risk of vascular compression on the nerve.

\section{REFERENCES}

1. Agrawal SM, Kambalimath DH: Trigeminal neuralgia involving supraorbital and infraorbital nerves. Natl J Maxillofacsurg 1:179-182, 2010

2. Akimoto H, Nagaoka T, Nariai T, Takada Y, Ohno K, Yoshino $\mathrm{N}$ : Preoperative evaluation of neurovascular compression in patients with trigeminal neuralgia by use of three-dimensional reconstruction from two types of high-resolution magnetic resonance imaging. Neurosurgery 51:956-961, 2002

3. Antonini G, Di Pasquale A, Cruccu G, Truini A, Morino S, Saltelli G, Romano A, Trasimeni G, Vanacore N, Bozzao A: Magnetic resonance imaging contribution for diagnostic symptomatic neurovascular contact in classical trigeminal neuralgia: A blinded case-control study and meta-analysis. Pain 155:1464-1471, 2014

4. Besta R, Shankar YU, Kumar A, Rajasekhar E, Prakash SB: MRI 3D CISS- a novel imaging modality in diagnostic trigeminal neuralgia - a review. J ClinDiagn Res 10(3):1-3, 2016

5. Blitz AM, Northcutt B, Shin J, Aygun N, Herzka DA, Theodros D, Goodwin CR, Lim M, Seeburg DP: Contrast enhanced ciss imaging for evaluation of neurovascular compression in trigeminal neuralgia: Improved correlation with symptoms and prediction of surgical outcomes. AJNR Am J Neuroradiol 39(9):1724-1732, 2018

6. Cha J, Kim ST, Kim HJ, Choi JW, Kim HJ, Jeon P, Kim KH, Byun HS, Park K: Trigeminal neuralgia: Assessment with T2 VISTA and FLAIR VISTA fusion imaging. Eur Radiol 21(12):26332639, 2011

7. Cheng J, Meng J, Liu W, Zhang H, Lei D, Hui X: Nerve atrophy and a small trigeminal pontine angle in primary trigeminal neuralgia: A morphometric magnetic resonance imaging study. World Neurosurg 104:575-580, 2017

8. Devor M, Govrin-Lippmann R, Rappaport ZH: Mechanism of trigeminal neuralgia: An ultrastructural analysis of trigeminal root specimens obtained during microvascular decompression surgery. J Neurosurg 96:532-543, 2002

9. Erbay SH, Bhadelia RA, O'Callaghan M, Gupta P, Riesenburger R, Krackov W, Polak JF: Nerve atrophy in severe trigeminal neuralgia: Noninvasive confirmation at MR imaging: Initial experience. Radiology 238:689-692, 2006

10. Garcia M, Naraghi R, Zumbrunn T, Rösch J, Hastreiter P, Dörfler A: High-resolution 3D-constructive interference in steadystate MR imaging and 3D time-of-flight MR angiography in neurovascular compression: A comparison between $3 \mathrm{~T}$ and 1.5 T. AJNR Am J Neuroradiol 33(7):1251-1256, 2012

11. Ha SM, Kim SH, Yoo EH, Han IB, Shin DA, Cho KG, Chung SS, Park YS: Patients with idiopathic trigeminal neuralgia have a sharper-than-normal trigeminal-pontine angle and trigeminal nerve atrophy. Acta Neurochir (Wien) 154:1627-1633, 2012 
Gunesli A. and Tufan K: MRI Findings in Trigeminal Neuralgia

12. Haller S, Etienne L, Kövari E, Varoquaux AD, Urbach $H$, Becker M: Imaging of neurovascular compression syndromes: Trigeminal neuralgia, hemifacial spasm, vestibular paroxysma and glossopharyngeal neuralgia. AJNR Am J Neuroradiol 37(8):1384-1392, 2016

13. Harsha KJ, Kesavadas C, Chinchure S, Thomas B, Jagtap $\mathrm{S}$ : Imaging of vascular causes of trigeminal neuralgia. J Neuroradiol 39:281-289, 2012

14. Horínek D, Brezová V, Nimsky C, Belsan T, Martinkovic L, Masopust V, Vrána J, Kozler P, Plas J, KrýsI D, Varjassyová A, Ghaly $\mathrm{Y}$, Benes V: The MRI volumetry of the posterior fossa and its substructures in trigeminal neuralgia: A validated study. Acta Neurochir (Wien) 151:669-675, 2009

15. Huges MA, Frederickson AM, Branstetter BF, Zhu X, Sekula RF Jr: MRI of the trigeminal nerve in patients with trigeminal neuralgia secondary to vascular compression. AJR Am J Roentgenol 206(3):595-600, 2016

16. Leal PR, Froment JC, Sindou M: MRI sequences for detection of neurovascular conflicts in patients with trigeminal neuralgia and predictive value for characterization of the conflict particularly degree of vascular compression. Neurochirurgie 56:43-49, 2010
17. Northcutt BG, Seeburg DP, Shin J, Aygun N, Herzka DA, Theodros D, Goodwin CR, Bettegowda C, Lim M, Blitz AM: High resolution MRI findings following trigeminal rhizotomy. AJNR Am J Neuroradiol 37(10):1920-1924, 2016

18. Parise $M$, Acioly MA, Ribeiro $C T$, Vincent $M$, Gasparetto EL: The role of the cerebellopontine angle cistern area and trigeminal nerve length in the pathogenesis of trigeminal neuralgia: A prospective case-control study. ActaNeurochir (Wien) 155:863-868, 2013

19. Park SH, Hwank SK, Lee SH, Park J, Hwank JH, Hamm IS: Nerve atrophy and a small cerebellopontine angle cistern in patients with trigeminal neuralgia. J Neurosurg 110:633-637, 2009

20. Pollock BE, Ecker RD: A prospective cost-effectiveness study of trigeminal neuralgia surgery. Clin J Pain 21:317-322, 2005

21. Rasche D, Kress B, Stippich C, Nennig E, Sartor K, Tronnier VM: Volumetric measurement of the pontomesencephalic cistern in patients with trigeminal neuralgia and healthy controls. Neurosurgery 59:614-620, 2006 